\title{
Apresentação da Técnica de Tomografia por Impedância Magnética Aplicada ao Imageamento em Medicina
}

\author{
Technic's Presentation of the Magnetic Impedance Tomography Applied to Medical Imaging
}

\author{
Helcimar Moura de Jesus e Thierry J. Lemaire* \\ Laboratório de Instrumentação - Departamento de Física - UEFS \\ Campus Universitário Km 03 BR 116 \\ Feira de Santana - BA - 44031-460
}

\begin{abstract}
A técnica de Tomografia por Impedância Magnética é apresentada de forma introdutória e o problema inverso associado, no caso de um corpo cilíndrico, é resolvido com o algoritmo Simulated Annealing. Os resultados apresentados neste artigo foram obtidos por um dos autores no seu trabalho de final de curso. Considerações práticas sobre a questão do ruído na instrumentação assim como o impacto da presença deste ruído nos sinais a ser tratados não são abordados.
\end{abstract}

Palavras-chaves: Tomografia, Condutividade, Impedância, Campo Magnético, Corrente Elétrica, Física Médica.

\begin{abstract}
The Magnetic Impedance Tomography technic's is presented at an introductory level and the associated inverse problem, for the cylindrical body, is solved with the simulated annealing algorithm. The results presented in this article have been obtained by one of the authors in his final work of graduation. Practical considerations about the problem of noise in instrumentation and the consequences of this noise in the treatment of the signal used to inversion, are not treated.
\end{abstract}

Key-words: Tomography, Conductivity, Impedance, Magnetic Field, Electrical Current, Medical Physics.

\section{INTRODUÇÃO}

O desenvolvimento de instrumentos destinados à realização de imagens de partes do corpo humano tem sido extremamente útil na medicina e tem permitido avanços importantes nesta área. Em particular, as técnicas de imageamento permitem emitir diagnósticos mais rapidamente e também, geralmente, sem ou com pouco trauma para o paciente. Podemos citar especialmente o avanço nesta área gerado pela descoberta dos raios-X em 1895 por Roentgen [1] e que teve um papel importante durante a primeira guerra mundial no tratamento dos soldados feridos. As técnicas de imageamento podem ser classificadas em dois grupos principais: - as técnicas invasivas, que necessitam de introdução de algum objeto como por exemplo uma fibra óptica para endoscopia, a injeção de algum líquido radioativo para a TEP (Tomografia por Emissão de Positrons), ou de sondas diversas no paciente; - as não invasivas, cujo princípio é baseado na medição da resposta de parte do corpo humano a algum sinal (ultra som, imagens por ressonância magnética nuclear, imagem ou tomografia de raios- $\mathrm{X}, \ldots)$; ou de sinais naturalmente emitidos pelo corpo (imageamento no infravermelho, eletroencefalograma, eletrocardiograma, ...).

Entretanto, as técnicas mais sofisticadas de imageamento $(R M N$, raios-X) [2] envolvem equipamentos custosos e relativamente pesados. De fato, o preço destes equipamentos pode variar de algumas dezenas de milhares até alguns milhões de reais. Existe, portanto, um número pequeno de equipamentos de alto custo disponíveis para atender a população. Como conseqüência, estes equipamentos são

*Endereço Eletrônico: tlemaire@uefs.br geralmente utilizados para tratar de um trauma ou de uma doença, fazendo com que exames de prevenção por exemplo para detecção de câncer, não sejam possíveis. Podemos também salientar uma outra limitação associada à algumas técnicas de imageamento tais como a tomografia computadorizada de raios-X (radiação ionizante) que são prejudiciais a saúde e portanto o número de sessões às quais um paciente poderia ser submetido durante sua vida deveria ser limitado.

Observamos então que é importante que outras técnicas de imageamento do corpo humano sejam desenvolvidas com menor custo e sem prejuízo à saúde, para aumentar o número de pessoas atendidas.

De fato, grupos de diversos países estão estudando novas técnicas de imageamento $[3,4]$. Em particular, uma família de técnicas envolve a medição (ou o mapeamento) da condutividade (bioimpedância elétrica) de certas regiões do corpo humano. Estas técnicas estão baseadas no fato de que cada tipo de tecido do corpo é caracterizado por uma resistividade e portanto pode ser reconhecido medindo esta característica física que varia numa larga escala (pelo menos 2 ordens de grandezas), comparado com por exemplo o coeficiente de absorção de raios-X por tecidos, que varia de aproximadamente de um fator 2 . As principais técnicas são a Tomografia por Impedância Elétrica (TIE) [5] e a Tomografia por Impedância Magnética [5, 6]. Basicamente, nestas duas técnicas, uma corrente elétrica senoidal de baixa intensidade é aplicada no paciente e o efeito (presença de uma tensão entre dois pontos determinados do corpo ou de um campo magnético na vizinhança do paciente) é medido e depois tratado para gerar um mapa de condutividade da região estudada. As suas principais características são o relativo baixo custo, a velocidade de aquisição dos dados, eventualmente o manuseio (equipamento que pode ser miniaturizado para monitoramento contínuo do paciente) e o fato de não envolver fenômenos prejudiciais à saúde, por- 
tanto, permitindo um número ilimitado de medições. Tipicamente, na tomografia por impedância magnética usa-se correntes com amplitudes de $100 \mathrm{~mA}$ nas freqüências de 16 $\mathrm{Hz}$ a $1 \mathrm{kHz}$. No entanto, numa escala de $10 \mathrm{kHz}$ a $1 \mathrm{MHz}$, os padrões de segurança europeus EN60601 (European Safety Standard EN60601) especificam que pode-se injetar com segurança uma corrente elétrica no corpo humano com amplitudes entre 1 e 10 mArms.

A seguir, descrevemos o princípio da Tomografia por Impedância Magnética (TIM) e alguns resultados de simulações obtidos por um dos autores [7] são apresentados.

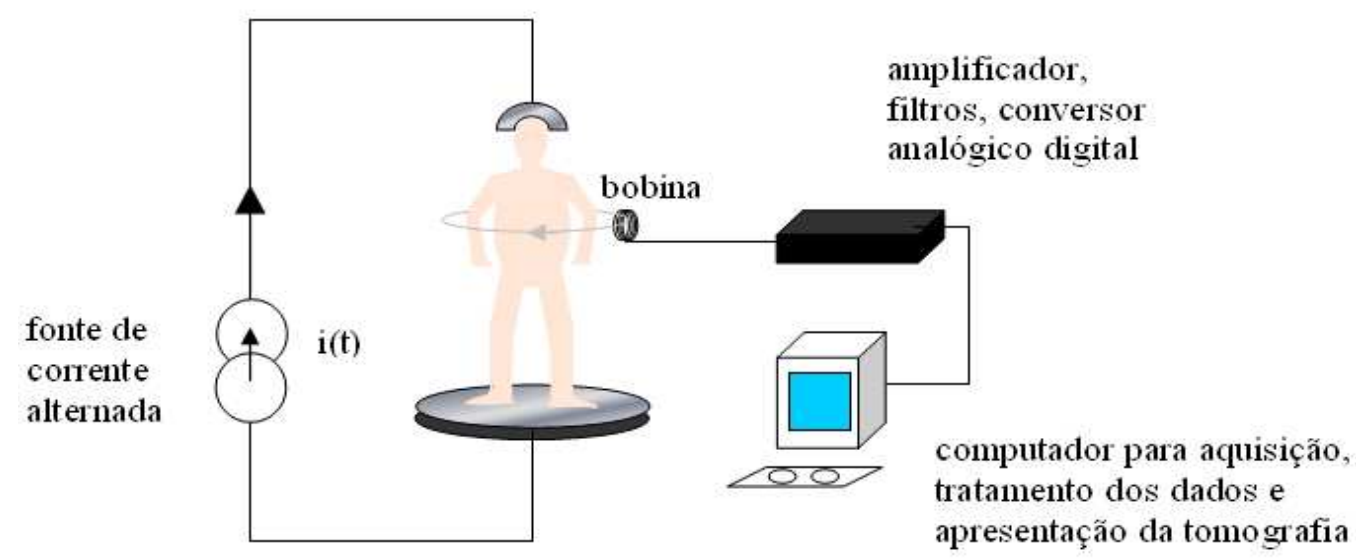

Fig. 1 - Equipamento utilizado para a TIM

\section{A TÉCNICA DE TOMOGRAFIA POR IMPEDÂNCIA MAGNÉTICA}

estudada.

\section{B. Um Modelo Associado à TIM}

\section{A. Princípio da TIM}

O princípio básico da técnica de Tomografia por Impedância Magnética é baseado na medida do campo magnético gerado por uma distribuição de corrente elétrica alternada, a determinar, dentro do corpo estudado. A corrente senoidal de baixa freqüência $(10 \mathrm{kHz}-1 \mathrm{MHz})$ e de baixa intensidade, por exemplo de 1 a $10 \mathrm{~mA}$ segundo as normas de segurança Européias EN60601, é aplicada ao corpo com dois eletrodos (ver Figura 1) cuja geometria e posicionamento não são tanto cruciais como no caso da TIE $[5]$.

Devido à corrente injetada no paciente, conforme prevê a Física [8], um campo magnético é gerado no espaço e é detectado com um sensor (bobina, sensor Hall, magnetoresistor, ...) em torno do paciente (num plano cuja intersecção com o corpo defina a região estudada). O sinal elétrico (geralmente uma tensão) recolhido do sensor é amplificado, filtrado, digitalizado e armazenado num computador que, em seguida, recebe os tratamentos adequados (inversão de dados) para se obter um mapa da condutividade da seção
A modelização do principal fenômeno físico requer o uso da teoria do eletromagnetismo. A lei de Biot-Savard [8] permite relacionar a densidade de corrente elétrica de condução $\vec{j}(\vec{r})$ presente num domínio $\Omega$ do espaço com o campo magnético, $\vec{B}(\vec{r})$, por ela gerado no ponto $\vec{r}$ (ver Figura $2)$,

$$
\vec{B}(\vec{r})=\frac{\mu_{0}}{4 \pi} \int_{\Omega} \frac{\vec{j}\left(\vec{r}^{\prime}\right) \times\left(\vec{r}-\vec{r}^{\prime}\right)}{\left\|\vec{r}-\vec{r}^{\prime}\right\|^{3}} d^{3} \vec{r}^{\prime},
$$

onde $\mu_{0}$ é a permeabilidade do vácuo (ou do ar). Esta expressão pode ser discretizada considerando no lugar de uma distribuição contínua de corrente, uma distribuição discreta $\left\{i_{k}\right\}$ de $N$ correntes lineares. Cada corrente $i_{k}$ que circula num circuito linear de comprimento $L$ gera um campo magnético (ver Figura 2 para as notações gerais):

$$
\vec{B}_{k}(\vec{r})=\frac{\mu_{0} i_{k}}{4 \pi} \int_{L / 2}^{L / 2} \frac{d \vec{s} \times(\vec{r}-\vec{s})}{\|\vec{r}-\vec{s}\|^{3}},
$$




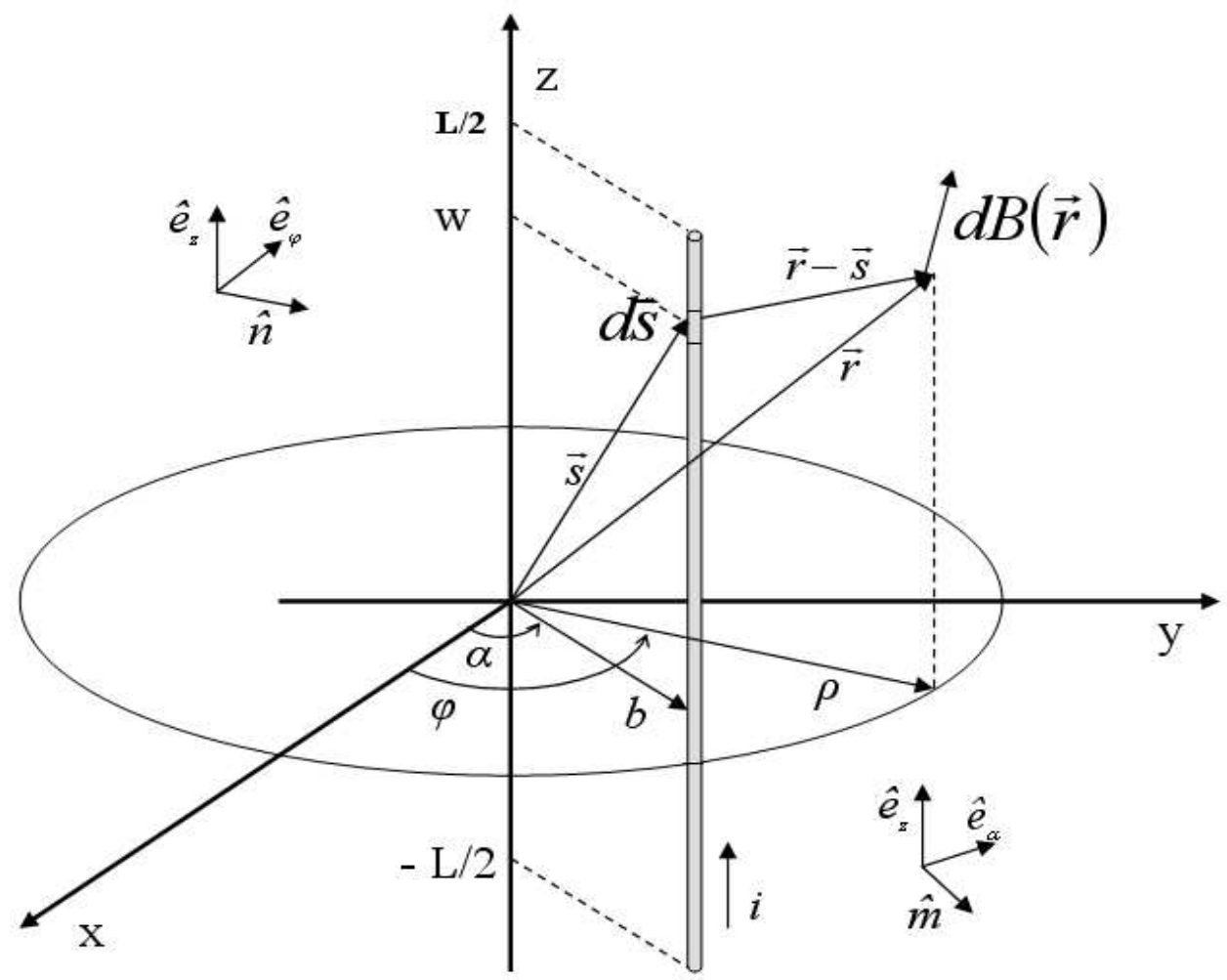

Fig. 2 - Definições das notações

O campo magnético total $\vec{B}(\vec{r})$ gerado por uma região discreta (ver Figura 3 mostrando um corpo constituído de $N$ circuitos lineares e paralelos) de comprimento $L$ escreve-se:

$$
\vec{B}(\vec{r})=\sum_{k=1}^{N} \vec{B}_{k}(\vec{r})
$$

onde,

$$
\begin{aligned}
\vec{B}_{k}(\vec{r}) & =\frac{\mu_{0} i_{k}}{4 \pi} f_{k}\left(\rho, \varphi, z, b_{k}, a_{k}\right) \times \\
& \times\left\{-b_{k} \sin \left(\varphi-a_{k}\right) \hat{n}+\left[\rho-b_{k} \cos \left(\varphi-a_{k}\right)\right] \hat{e}_{\varphi}\right\}
\end{aligned}
$$

e

$$
\begin{aligned}
& f_{k}\left(\rho, \varphi, z, b_{k}, a_{k}\right) \\
= & \int_{L / 2}^{L / 2} \frac{d w}{\left[\rho^{2}+b_{k}^{2}-2 \rho \cos \left(\varphi-a_{k}\right)+(z-w)^{2}\right]^{3 / 2}} .
\end{aligned}
$$

Observamos que nas equações acima, a contribuição ao campo magnético da fiação ligando a fonte de corrente ao paciente não é levada em conta porque ela pode ser removida do campo magnético medido, por cálculo. Consideremos portanto somente o campo magnético gerado pela distribuição de corrente dentro do corpo estudado.

Finalmente, caso seja utilizado uma bobina de $P$ espiras e de área orientada $\vec{A}$ para detectar o campo magnético, a força eletromotriz (f.e.m.) induzida é dada pela lei de Faraday que pode ser simplificada, para bobina pequenas o suficiente (para que o campo magnético sensoriado por esta, $\vec{B}_{\text {bobina }}$, seja aproximadamente uniforme):

$$
\zeta=-P \vec{A} \cdot \frac{d}{d t} \vec{B}_{b o b i n a}
$$

\section{Simulações Numéricas}

A fim de ilustrar o comportamento do campo magnético gerado por uma dada distribuição de corrente, e fixar ordens de grandezas, consideremos uma distribuição de 9 correntes (descrita pela Figura 3) caracterizada pela Tabela I. Os dados que caracterizam o sistema são resumidos na Tabela II. As f.e.m. 's $\zeta_{\varphi}$ e $\zeta_{n}$ calculadas para $P=1$ correspondem às componentes $\vec{B}_{\varphi}$ e $\vec{B}_{n}$ (ver Figura 2 ).

Observamos que as f.e.m.'s induzidas no sensor devido a correntes de alguns mA, são bastante fracas (da ordem do $\mu \mathrm{V}$ ), o que pode representar algumas dificuldades para medições em ambiente ruidoso. Entretanto, lembramos que estas f.e.m.'s podem ser amplificadas, aumentando o número de espiras do sensor. Salientamos também que existem outros sensores de campo magnético que podem ser mais adaptados para o problema mas não iremos discutir este aspecto prático (mas importante) da instrumentação.

Observamos que os "picos" que aparecem na curva da Figura 4 relativo a $\zeta_{\varphi}$ correspondem à proximidade de uma linha percorrida por uma corrente relativamente elevada (comparada com as outras) e portanto a uma região de alta condutividade. 


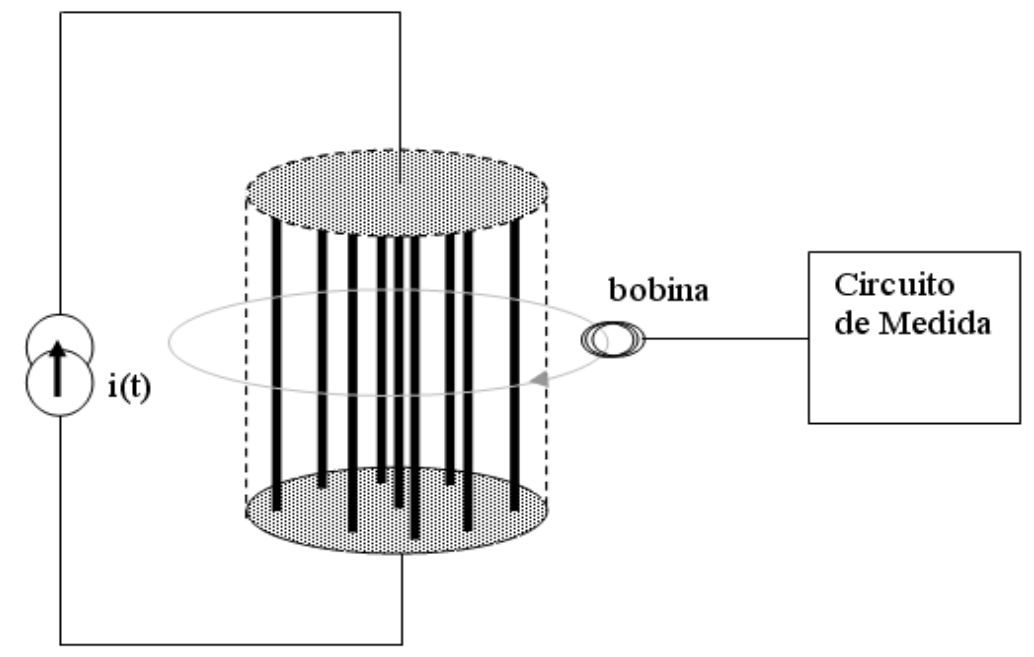

Fig. 3 - Distribuição discreta de corrente equivalente ao corpo estudado

\section{PROBLEMA INVERSO ASSOCIADO À TIM}

\section{A. Considerações gerais}

As f.e.m. 's mensuradas pelo instrumento para os diversos ângulos $\phi$ são, como mostram as relações (3) até (6), funções lineares das correntes $\left\{i_{k}\right\}$. Isto significa que podemos escrever o conjunto de $(M)$ f.e.m. 's medidas em função das correntes numa forma matricial:

$$
\vec{\zeta}=\Xi \vec{i}
$$

onde a matriz $\Xi$ é obtida empregando as relações (3-6) acima citadas e,

$$
\begin{aligned}
\vec{\zeta} & =\left(\zeta_{\phi}\left(\phi_{1}\right) \zeta_{n}\left(\phi_{1}\right), \ldots, \zeta_{\phi}\left(\phi_{M}\right), \zeta_{n}\left(\phi_{M}\right)\right)^{T} \\
\mathrm{e} \quad \vec{i} & =\left(i_{1}, \ldots, i_{N}\right)^{T}
\end{aligned}
$$

Entretanto, apesar da simplicidade das equações envolvidas, a equação (7) é mal condicionada. Isto significa que não é possível inverter esta relação simplesmente, mesmo quando a matriz $\Xi$ é quadrada, e obter um resultado único. Para determinar a distribuição de corrente dentro do corpo, utilizamos um algoritmo de otimização chamado Simulated Annealing [9]. Este técnica de busca aleatória é baseada na analogia com o fenômeno de recozimento térmico empregado em metalurgia para obter aços de propriedades mecânicas bem definidas. O algoritmo pode ser resumido pelo pseudo-programa a seguir, considerando o problema de minimização de uma função custo $C(\vec{x})$ cujos parâmetros $\vec{x}$ devem ser otimizados:

Etapa 1 : fixação de $\vec{x}_{0}$ (primeira determinação de $\vec{x}$ );

Etapa 2 : geração de um vizinho $\vec{x}_{\nu}$ de $\vec{x}_{0}$ com um mecanismo envolvendo um gerador de números aleatórios;

Etapa 3 : aplicação do critério de Metrópolis, i.e., sendo $\triangle C=C\left(\vec{x}_{\nu}\right)-C\left(\vec{x}_{0}\right):$ se $\triangle C \leq 0$ a configuração vizinha é aceita, se não, a probabilidade de aceitação é dada por: $\exp (-\triangle C / T)$, onde $T$ é um parâmetro de controle sobre a convergência do processo (com papel análogo ao da temperatura no caso do recozimento térmico);

Etapa 4: aplicação de uma mudança de temperatura seguindo uma determinada função;

Etapa 5 : aplicação de um critério de convergência. Se este não for verificado, volta-se à etapa 2 (caso $\vec{x}_{\nu}$ for aceito, $\vec{x}_{0}$ torna-se $\vec{x}_{\nu}$ ). Caso contrário o algoritmo deve parar.

Na prática, a fim de restringir o espaço de soluções do problema, acrescentamos duas condições (restrições). A primeira impõe o valor conhecido da corrente total $i_{\text {tot }}$ injetada no corpo estudado e a segunda considera somente as amplitudes positivas das correntes $i_{k}$ :

$$
\begin{array}{r}
i_{\text {tot }}=i_{1}+\ldots+i_{N}, \\
i_{k} \geq 0, \quad k=1, \ldots, N .
\end{array}
$$

Para implementar este método, definimos uma função custo (ou objetivo) que deve ser minimizada:

$$
C(\vec{i})=\frac{1}{M} \sum_{j=1}^{M}\left[\frac{\zeta^{e x p}\left(\rho, \phi_{j}, z\right)-\zeta^{e s t}\left(\rho, \phi_{j}, z ; \vec{i}\right)}{\zeta^{\exp }\left(\rho, \phi_{j}, z\right)}\right]^{2}
$$

As variáveis $\zeta^{\exp }\left(\rho, \phi_{j}, z\right)$ (resp. $\left.\zeta^{e s t}\left(\rho, \phi_{j}, z ; \vec{i}\right)\right)$ são as f.e.m.'s medidas (resp. estimadas a partir da corrente $\vec{i}$ ). De fato, nas simulações realizadas, utilizamos somente as f.e.m. 's $\zeta_{\phi}$, na função custo (ver [7]), considerando que há informações suficientes neste "sinal" para construir uma tomografia da região estuda do corpo. Definimos também um estimador do erro sobre a determinação das correntes para as tentativas de inversão com dados artificiais (salientamos que esta quantidade não é acessível com problemas reais de TIM porque a distribuição de corrente, $\vec{i}^{\text {exato }}$, não é conhecida): 


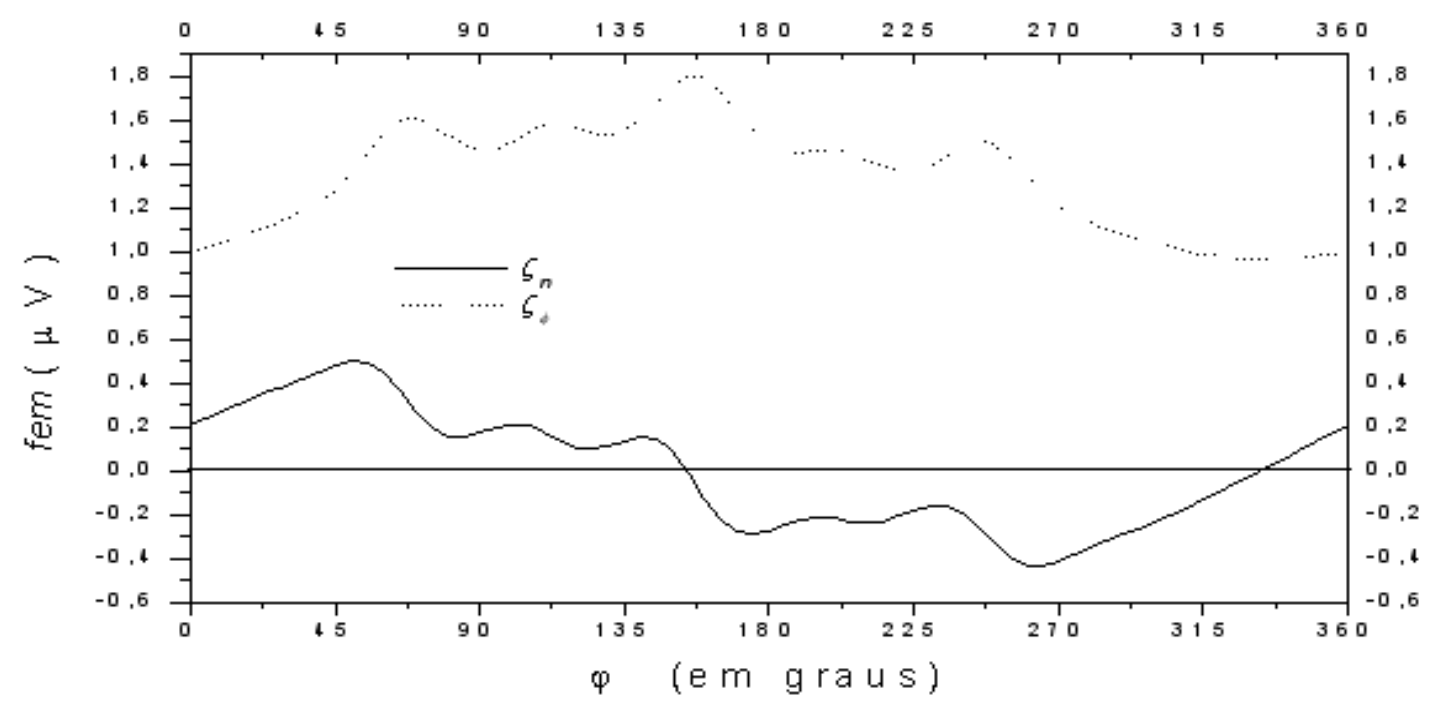

Fig. $4-\zeta_{\varphi}$ e $\zeta_{n}$ calculadas para a distribuição de corrente da Figura 3 e nas condições descritas nas Tabela I e II

$$
\delta=\frac{1}{N} \frac{\left\|\vec{i}^{\text {exato }}-\vec{i}\right\|^{2}}{\| \overrightarrow{i^{\text {exato }} \|^{2}}},
$$

No parágrafo seguinte, mostramos um exemplo de inversão a fim de ilustrar a eficácia do método de inversão acima exposto.

\section{B. Resultados numéricos de inversão para um modelo simples}

Consideremos o corpo (cilíndrico) modelado com 9 correntes (ver Figura 3). Dados sintéticos foram gerados com um programa FORTRAN [7] e depois invertidos com o Simulated Annealing. Os valores do campo magnético são obtidos a uma distância $\rho=9 \mathrm{~cm}$ para $\theta=0^{0}, 5^{0}, 10^{0}, \ldots, 355^{0}$ dando um total de $M=72$ medidas. Os parâmetros que definem as linhas de corrente são:

$$
\begin{aligned}
b_{1} & =0,0 \mathrm{~cm} \\
b_{k} & =6,0 \mathrm{~cm} k=2, \ldots, 9 \\
\text { e } \alpha_{k} & =\left(k-\frac{1}{2}\right) \frac{\pi}{4} \quad \operatorname{rad}, \quad k=1, \ldots, 9 .
\end{aligned}
$$

Os outros parâmetros do problema são descritos na Tabela II. Um resultado é apresentado na Tabela III: observamos que 5 das 9 correntes reconstruídas são relativamente próximas dos valores verdadeiros (erro relativo inferior a $20 \%$ ), e em particular quando estas correntes são relativamente altas. As f.e.m. 's $\zeta_{\phi}$ associadas às correntes verdadeiras e reconstruídas são mostradas na Figura 5. Podemos observar que elas são muito similares, mostrando que a inversão de dados ocorreu satisfatoriamente, o que corresponde a uma função custo assim como um estimador sobre a determinação das correntes relativamente pequenos. No processo de reconstrução das correntes, o algoritmo realizou 15000 iterações processadas em um tempo de aproximadamente 68 segundos, com um Pentium 4 de $2.8 \mathrm{GHz}$. O sistema iniciou com um parâmetro de controle elevado, $T_{0}=10^{6}$, o qual foi decaindo de acordo com a função: $T_{n}=\beta T_{n-1}$ onde a constante $\beta$, que deve estar compreendida entre zero e um, teve o valor $\beta=0,8$. A fim de que o sistema evoluísse em equilíbrio térmico, foram feitas 60 iterações para cada valor de $T_{n}$. Seguindo essa função de decaimento, o valor final de $T$ é da ordem de $10^{-18}$. Os valores dos parâmetros aqui apresentados são os resultados de diversas tentativas de resolução do nosso problema de otimização, e basicamente foram obtidos de forma empírica.

\section{CONCLUSÕES}

Neste artigo foi apresentada de forma sucinta a técnica de imageamento chamada Tomografia por Impedância Magnética. Esta técnica, recentemente introduzida, representa certas vantagens em relação aos métodos convencionais: ela é mais barata, rápida e não apresenta nenhum perigo para o paciente. Entretanto, precisa ainda muito trabalho de pesquisa para aprimorar esta técnica que não permite atualmente obter imagens com boa resolução. Os resultados apresentados (obtidos durante um trabalho de final de curso de graduação [7]) permitiram ilustrar de forma simples o problema inverso associado, mas não apresentam interesses práticos por conta da resolução muito baixa utilizada no modelo. Uma forma de melhorar a resolução implica na utilização de mais domínios para representar o objeto. Também existem técnicas de otimização de maior eficácia que podem ser utilizadas (por exemplo o "Generalized Simulated Annealing" ou métodos híbridos). 


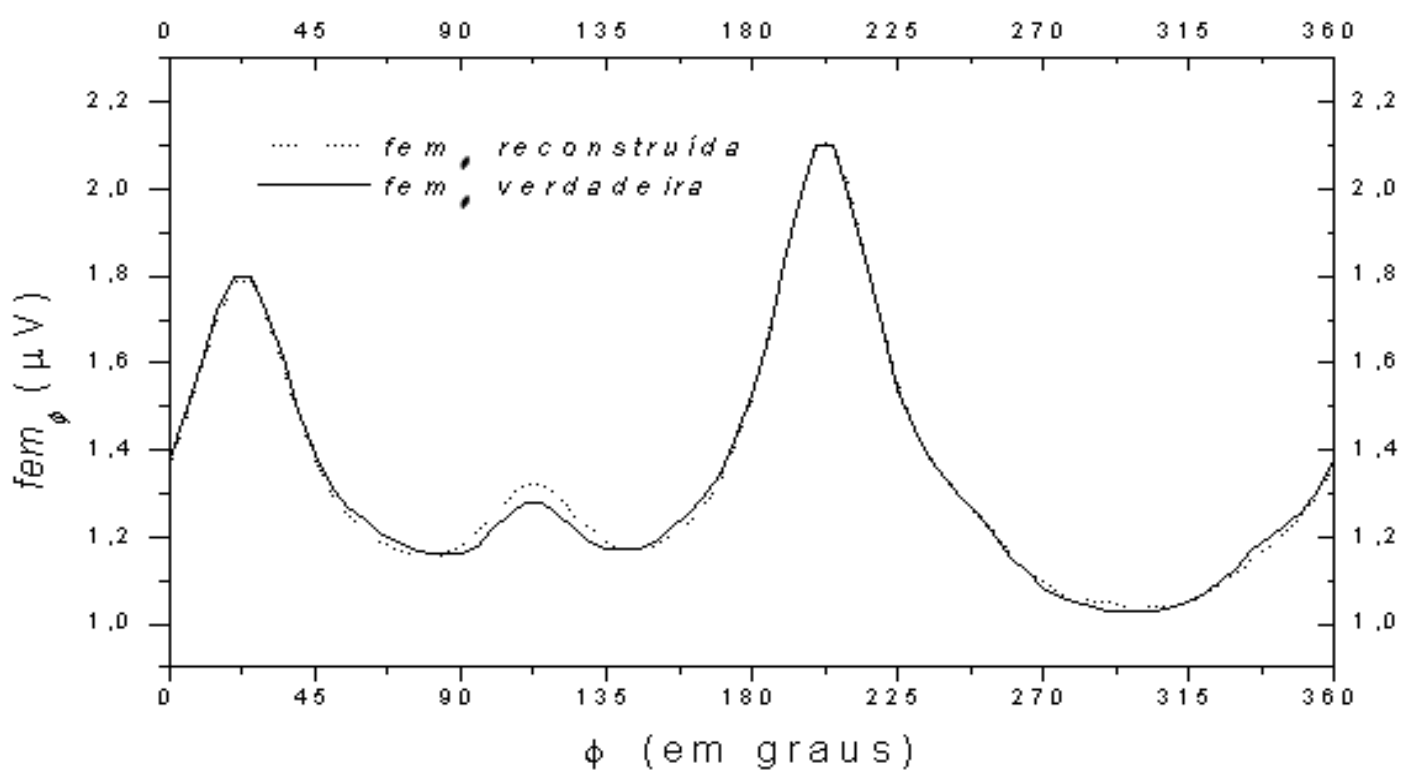

Fig. 5 - Comparação entre a força eletromotriz induzida pelas correntes verdadeiras e reconstruídas

\begin{tabular}{|c|c|c|c|c|}
\hline \multirow{2}{*}{ Região $\boldsymbol{k}$} & \multicolumn{3}{|c|}{ coordenadas } & \multirow{2}{*}{$\begin{array}{c}\text { Correntes } \\
\boldsymbol{i}_{\boldsymbol{k}}(\mathrm{m} A)\end{array}$} \\
\cline { 2 - 4 } $\mathbf{\mathbf { b }}(\mathbf{c m})$ & $\boldsymbol{\alpha}(\mathbf{g r a u s})$ & $\mathbf{z}(\mathbf{c m})$ & 1,09 \\
\hline $\mathbf{0}$ & 0,0 & 0,0 & 0,0 & 0,24 \\
\hline $\mathbf{1}$ & 6,0 & 22,5 & 0,0 & 1,98 \\
\hline $\mathbf{3}$ & 6,0 & 67,5 & 0,0 & 1,38 \\
\hline $\mathbf{4}$ & 6,0 & 112,5 & 0,0 & 2,43 \\
\hline $\mathbf{5}$ & 6,0 & 157,5 & 0,0 & 1,00 \\
\hline $\mathbf{6}$ & 6,0 & 202,5 & 0,0 & 1,71 \\
\hline 7 & 6,0 & 247,5 & 0,0 & 0,17 \\
\hline $\mathbf{8}$ & 6,0 & 229,5 & 0,0 & 0,00 \\
\hline
\end{tabular}

Tab. I - Distribuição das correntes

\begin{tabular}{|l|l|}
\hline Comprimento dos fios & $2 \mathrm{~m}$ \\
\hline Freqüência do sinal injetado & $100 \mathrm{kHz}$ \\
\hline Área da bobina & $1 \mathrm{~cm}^{2}$ \\
\hline Amplitude da corrente total & $10 \mathrm{~mA}$ \\
\hline Plano de medição & $\mathrm{z}=0$ \\
\hline
\end{tabular}

Tab. II - Parâmetros caracterizando o sistema de coleta de dados e o sistema estudado 


\begin{tabular}{|c|c|c|}
\hline Regiões k & $\begin{array}{c}\text { Correntes verdadeiras } \\
\text { (mA) }\end{array}$ & $\begin{array}{c}\text { Correntes reconstruídas } \\
(\mathrm{m} A)\end{array}$ \\
\hline $\mathbf{0}$ & 0,50 & 0,79 \\
\hline $\mathbf{1}$ & 3,00 & 2,94 \\
\hline $\mathbf{2}$ & 0,30 & 0,16 \\
\hline $\mathbf{4}$ & 1,00 & 1,17 \\
\hline $\mathbf{5}$ & 0,20 & 0,06 \\
\hline $\mathbf{6}$ & 4,00 & 4,00 \\
\hline 7 & 0,50 & 0,47 \\
\hline $\mathbf{8}$ & 0,10 & 0,12 \\
\hline 0,40 & 0,29 \\
\hline$C_{\min }=1,85.10^{-4}$ & \multicolumn{2}{|l}{} \\
\hline$\delta=6,87.10^{-4}$ & \multicolumn{2}{|l}{} \\
\hline
\end{tabular}

Tab. III - Comparação entre as correntes verdadeiras e reconstruídas

[1] R.M. Eisberg; Fundamentos da Física Moderna, Ed. Guanabara Dois S.A., RJ, (1979).

[2] D.J. Paul; Foresight Exploiting the Electromagnetic Spectrum project - Picturing people: non-destructive imaging, www.foresight.gov.uk.

[3] A.K. Louis; Medical imaging: state of the art and future development, Inverse Problems 8, 709 (1992).

[4] P.L Carson et al; Biomedical Imaging Research Opportunity Workshop: Report and Recommendations, Radiology 229 (2), 328 (2003).

[5] P.J. Riu, J. Rosell, R. Bragós, O. Casas; Electrical Bioimpedance Methods, Annals of the New York Academy of Sciences 873, NY, (1999).
[6] J.C. Tozer, R.H. Ireland, D.C. Barber; \& A.T. Barker, Electrical Bioimpedance Methods. Magnetic Impedance Tomography, Annals of the New York Academy of Sciences 873 353, NY, (1999).

[7] H. Moura de Jesus; Métodos de otimização aplicados a tomografia por impedância magnética, Trabalho de final de curso, Licenciatura em Física - UEFS, Feira de Santana BA, (2005).

[8] J.R. Reitz, F.J. Milford, W.C. Robert; Fundamentos da teoria eletromagnética, Ed. Campos, RJ (1982).

[9] P.J.V. Laarhoven, E.H. Aarts; Simulated Annealing: Theory and Applications, Reidel, Dordrecht (1987). 